Commun. Fac. Sci. Univ. Ank. Ser. A1 Math. Stat.

Volume 68, Number 2, Pages 1841-1851(2019)

DOI: $10.31801 /$ cfsuasmas.471397

ISSN 1303-5991 E-ISSN 2618-6470

http://communications.science.ankara.edu.tr/index.php?series=A1

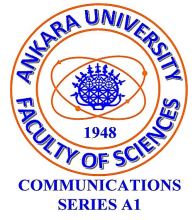

\title{
ON SOME NEW INEQUALITIES OF HERMITE HADAMARD TYPES FOR HYPERBOLIC $p$-CONVEX FUNCTIONS
}

\author{
NASHAT FARIED, MOHAMED S. S. ALI, AND ZEINAB M. YEHIA
}

\begin{abstract}
In this paper, we show that the power function $f^{n}(x)$ is hyperbolic $p$-convex function. Furthermore, we establish some new integral inequalities for higher powers of hyperbolic $p$-convex functions. Also, some applications for special means are provided as well.
\end{abstract}

\section{INTRODUCTION}

Let $f: I \rightarrow \mathbb{R}$ be a convex function on the interval $I$ of real numbers and $a, b \in I$ with $a<b$. There are many generalizations of the notion of convex functions see 2, 3, 5, 6. One way to generalize the notion of convex function is to replace linear functions by another family of functions in the sense of Beckenbach [2. In this paper, we deal with a family of hyperbolic functions

$$
H(x)=A \cosh p x+B \sinh p x,
$$

where $A, B$ arbitrary constants and $p \in \mathbb{R} \backslash\{0\}$.

The Hermite-Hadamard integral inequality for convex functions $f:[a, b] \rightarrow \mathbb{R}$

$$
f\left(\frac{a+b}{2}\right) \leq \frac{1}{b-a} \int_{a}^{b} f(x) d x \leq \frac{f(a)+f(b)}{2}
$$

is well known in the literature and has many applications for special means, see for example [7, 8, 9]. This inequality 1 was extended for hyperbolic $p$-convex functions in 1 as

$$
\frac{2}{p} f\left(\frac{a+b}{2}\right) \sinh p\left(\frac{b-a}{2}\right) \leq \int_{a}^{b} f(x) d x \leq \frac{1}{p}[f(a)+f(b)] \tanh p\left(\frac{b-a}{2}\right) .
$$

In current work, we proved that the higher powers of $\mathrm{f}(\mathrm{x})$ is hyperbolic $p$-convex function in addition to establish some new integral inequalities for higher powers of hyperbolic $p$-convex functions.

Received by the editors: October 16, 2018; Accepted: March 26, 2019.

2010 Mathematics Subject Classification. 26A51, 26B25, 26D15.

Key words and phrases. Generalized convex functions, hyperbolic $p$-convex functions, supporting functions, Hadamard's inequality.

(C)2019 Ankara University Communications Faculty of Sciences University of Ankara-Series A1 Mathematics and Statistics 


\section{Definitions and Preliminary Results}

In this section, we introduce the basic definitions and results which will be used later. For more informations see [1, 4], 10].

Definition 1. A function $f: I \rightarrow \mathbb{R}$ is said to be sub $H$-function on $I$ or hyperbolic $p$-convex function, if for any arbitrary closed subinterval $[u, v]$ of I the graph of $f(x)$ for $x \in[u, v]$ lies nowhere above the function, determined by the equation:

$H(x)=H(x, u, v, f)=A \cosh p x+B \sinh p x ; \quad p \in \mathbb{R} \backslash\{0\}$ where $A$ and $B$ are chosen such that $H(u)=f(u)$, and $H(v)=f(v)$.

Equivalently, for all $x \in[u, v]$

$$
f(x) \leq H(x)=\frac{f(u) \sinh p(v-x)+f(v) \sinh p(x-u)}{\sinh p(v-u)} .
$$

Remark 2. The hyperbolic p-convex functions possess a number of properties analogous to those of convex functions. For example: If $f: I \rightarrow \mathbb{R}$ is hyperbolic $p$-convex function, then for any $u, v \in I$, the inequality $f(x) \geq H(x)$ holds outside the interval $[u, v]$.

Definition 3. Let a function $f: I \rightarrow \mathbb{R}$ be hyperbolic p-convex function

$S_{u}(x)=A \cosh p x+B \sinh p x$

is said to be supporting function for $f(x)$ at the point $u \in(a, b)$ if

(1) $S_{u}(u)=f(u)$

(2) $S_{u}(x) \leq f(x) \forall x \in I$.

That is, if $f(x)$ and $S_{u}(x)$ agree at $x=u$ the graph of $f(x)$ does not lie under the support curve.

Proposition 4. If $f: I \rightarrow \mathbb{R}$ is a differentiable hyperbolic p-convex function, then the supporting function for $f(x)$ at the point $u \in I$ has the form

$$
S_{u}(x)=f(u) \cosh p(x-u)+\frac{f^{\prime}(u)}{p} \sinh p(x-u) .
$$

Theorem 5. Let $f: I \rightarrow \mathbb{R}$ be a two times continuously differentiable function. Then $f$ is hyperbolic p-convex function on $I$ if and only if $f^{\prime \prime 2} f(x) \geq 0$ for all $x$ in $I$.

Example 6. Let $f_{s}:(0, \infty) \rightarrow(0, \infty), f_{s}(x)=x^{s}$ with $p \in \mathbb{R} \backslash\{0\}$. If $s \in$ $(-\infty, 0) \cup[1, \infty)$ and

$$
f_{s}^{\prime \prime}(x)-p^{2} f_{s}(x)=s(s-1) x^{s-2}-p^{2} x^{s}=p^{2} x^{s-2}\left(\frac{s(s-1)}{p^{2}}-x^{2}\right) .
$$

Then,

$$
f_{s}^{\prime \prime}(x)-p^{2} f_{s}(x) \geq 0 \text { for } x \in\left(0, \frac{\sqrt{s(s-1)}}{|p|}\right)
$$


Hence, the power function $f_{s}$ for $s \in(-\infty, 0) \cup[1, \infty)$ is hyperbolic $p$-convex function on $\left(0, \frac{\sqrt{s(s-1)}}{|p|}\right)$.

Theorem 7. A function $f: I \rightarrow \mathbb{R}$ is hyperbolic p-convex function on $I$ if and only if there exist a supporting function for $f(x)$ at each point $x \in I$.

\section{Main Results}

Theorem 8. Let $f: I \rightarrow \mathbb{R}$ be non-negative, two times continuously differentiable and hyperbolic p-convex functions then the higher powers of $f(x)$ is hyperbolic $p$ convex function.

Proof. Since, $f(x)$ be non-negative and hyperbolic $p$-convex function, then using Theorem 5, we get

$$
f(x) \geq 0 \text { and } f^{\prime \prime 2} f(x) \geq 0 \forall x \in I .
$$

Hence,

$$
\begin{gathered}
f^{\prime \prime 2} f(x) \geq \frac{p^{2}}{n} f(x) \forall n \in \mathbb{N} . \\
\left(f^{n}(x)\right)^{\prime n-1}(x) f^{\prime}(x) \\
\left(f^{n}(x)\right)^{\prime \prime n-2}(x)\left(f^{\prime 2}+n f^{n-1}(x) f^{\prime \prime}(x)\right. \\
\left(f^{n}(x)\right)^{\prime \prime 2} f^{n}(x)=n(n-1) f^{n-2}(x)\left(f^{\prime 2}+n f^{n-1}(x) f^{\prime \prime 2} f^{n}(x)\right. \\
=n(n-1) f^{n-2}(x)\left(f^{\prime 2}+n f^{n-1}(x)\left(f^{\prime \prime}(x)-\frac{p^{2}}{n} f(x)\right) .\right.
\end{gathered}
$$

Now using (4), (5) we conclude that

$$
\left(f^{n}(x)\right)^{\prime \prime 2} f^{n}(x) \geq 0 .
$$

Hence, $f^{n}(x)$ is hyperbolic $p$-convex function $\forall n \in \mathbb{N}$.

Theorem 9. Let $f: I \rightarrow \mathbb{R}$ be a non-negative hyperbolic p-convex function, $n \in \mathbb{N}$, and $a, b \in I$ with $a<b$, Then

$$
\int_{a}^{b} f^{n}(x) d x \leq \sinh ^{-n} p(b-a) \sum_{r=0}^{n} \frac{1}{\mu}\left(\begin{array}{l}
n \\
r
\end{array}\right)[f(a)]^{n-r}[f(b)]^{r}\left[e^{\mu b+\lambda}-e^{\mu a+\lambda}\right],
$$

where $\lambda=p b(n-r)$ - arp, and $\mu=(2 r-n) p$.

Proof. Since, $f(x)$ is hyperbolic $p$-convex function, then from Definition 1 we have

$$
f(x) \leq H(x) \quad \forall x \in[a, b] .
$$

As $\mathrm{f}(\mathrm{x})$ is non-negative, we get:

$$
f^{n}(x) \leq H^{n}(x) \quad \forall n \in \mathbb{N}
$$


Thus, using (2), one obtains

$$
\begin{aligned}
& \int_{a}^{b} f^{n}(x) d x \leq \int_{a}^{b} H^{n}(x) d x \\
& =\frac{1}{\sinh ^{n} p(b-a)} \int_{a}^{b}[f(a) \sinh p(b-x)+f(b) \sinh p(x-a)]^{n} d x \\
& =\sinh ^{-n} p(b-a) \sum_{r=0}^{n}\left(\begin{array}{l}
n \\
r
\end{array}\right)[f(a)]^{n-r}[f(b)]^{r} \int_{a}^{b} \sinh ^{r} p(x-a) \sinh ^{n-r} p(b-x) d x \\
& =\sinh ^{-n} p(b-a) \sum_{r=0}^{n}\left(\begin{array}{l}
n \\
r
\end{array}\right)[f(a)]^{n-r}[f(b)]^{r} \\
& \times \int_{a}^{b}\left[\frac{e^{p(x-a)}-e^{-p(x-a)}}{2}\right]^{r}\left[\frac{e^{p(b-x)}-e^{-p(b-x)}}{2}\right]^{n-r} d x \\
& \leq \sinh ^{-n} p(b-a) \sum_{r=0}^{n}\left(\begin{array}{l}
n \\
r
\end{array}\right)[f(a)]^{n-r}[f(b)]^{r} \int_{a}^{b} e^{r p(x-a)} e^{p(n-r)(b-x)} d x \\
& =\sinh ^{-n} p(b-a) \sum_{r=0}^{n}\left(\begin{array}{l}
n \\
r
\end{array}\right)[f(a)]^{n-r}[f(b)]^{r} \int_{a}^{b} e^{r p(x-a)+p(n-r)(b-x)} d x \\
& =\sinh ^{-n} p(b-a) \sum_{r=0}^{n}\left(\begin{array}{l}
n \\
r
\end{array}\right)[f(a)]^{n-r}[f(b)]^{r} \int_{a}^{b} e^{p(2 r-n) x+p b(n-r)-a r p} d x \\
& =\sinh ^{-n} p(b-a) \sum_{r=0}^{n} \frac{1}{\mu}\left(\begin{array}{l}
n \\
r
\end{array}\right)[f(a)]^{n-r}[f(b)]^{r}\left[e^{\mu b+\lambda}-e^{\mu a+\lambda}\right],
\end{aligned}
$$

where $\lambda=p b(n-r)-a r p$, and $\mu=(2 r-n) p$.

Hence, the theorem follows.

Theorem 10. Let $f: I \rightarrow \mathbb{R}$ be a differentiable hyperbolic $p$-convex function, $n \in \mathbb{N}$, and $a, b \in I$ with $a<b$, Then

$$
\int_{a}^{b} f^{2 n-1}(x) d x \geq \sum_{r=0}^{2 n-1}\left(\begin{array}{c}
2 n-1 \\
r
\end{array}\right) f^{2 n-r-1}(a)\left(\frac{f^{\prime}(a)}{p}\right)^{r} \sum_{k=0}^{r} \frac{(-1)^{k}}{2^{r} \alpha}\left(\begin{array}{l}
r \\
k
\end{array}\right)\left[e^{\alpha b+\beta}-e^{\alpha a+\beta}\right],
$$

where $\alpha=p(r-2 k)$, and $\beta=a p(2 k-r)$.

Proof. Since, $f(x)$ is hyperbolic $p$-convex function, then from Definition 3 , we have

$$
f(x) \geq S_{a}(x) \forall x \in I
$$

and consequently,

$$
f^{2 n-1}(x) \geq S_{a}^{2 n-1}(x) \quad \forall n \in \mathbb{N}
$$


Thus, using (3) and $\cosh p(x-a) \geq 1$, one has

$$
\begin{aligned}
\int_{a}^{b} f^{2 n-1}(x) d x \geq \int_{a}^{b} S_{a}^{2 n-1}(x) d x \\
=\int_{a}^{b}\left[f(a) \cosh p(x-a)+\frac{f^{\prime}(a)}{p} \sinh p(x-a)\right]^{2 n-1} d x \\
=\sum_{r=0}^{2 n-1}\left(\begin{array}{c}
2 n-1 \\
r
\end{array}\right) f^{2 n-r-1}(a)\left(\frac{f^{\prime}(a)}{p}\right)^{r} \int_{a}^{b} \cosh ^{2 n-r-1} p(x-a) \sinh ^{r} p(x-a) d x \\
\geq \sum_{r=0}^{2 n-1}\left(\begin{array}{c}
2 n-1 \\
r
\end{array}\right) f^{2 n-r-1}(a)\left(\frac{f^{\prime}(a)}{p}\right)^{r} \int_{a}^{b} \sinh ^{r} p(x-a) d x \\
=\sum_{r=0}^{2 n-1}\left(\begin{array}{c}
2 n-1 \\
r
\end{array}\right) f^{2 n-r-1}(a)\left(\frac{f^{\prime}(a)}{p}\right)^{r} \int_{a}^{b}\left[\frac{e^{p(x-a)}-e^{-p(x-a)}}{2}\right]^{r} d x \\
=\sum_{r=0}^{2 n-1}\left(\begin{array}{c}
2 n-1 \\
r
\end{array}\right) f^{2 n-r-1}(a)\left(\frac{f^{\prime}(a)}{p}\right)^{r} \int_{a}^{b} \sum_{k=0}^{r} \frac{(-1)^{k}}{2^{r}}\left(\begin{array}{l}
r \\
k
\end{array}\right) e^{p(r-k)(x-a)} e^{-p k(x-a)} d x \\
=\sum_{r=0}^{2 n-1}\left(\begin{array}{c}
2 n-1 \\
r
\end{array}\right) f^{2 n-r-1}(a)\left(\frac{f^{\prime}(a)}{p}\right)^{r} \sum_{k=0}^{r} \frac{(-1)^{k}}{2^{r}}\left(\begin{array}{l}
r \\
k
\end{array}\right) \int_{a}^{b} e^{p(r-2 k) x+a p(2 k-r)} \\
=\sum_{r=0}^{2 n-1}\left(\begin{array}{c}
2 n-1 \\
r
\end{array}\right) f^{2 n-r-1}(a)\left(\frac{f^{\prime}(a)}{p}\right)^{r} \sum_{k=0}^{r} \frac{(-1)^{k}}{2^{r} \alpha}\left(\begin{array}{l}
r \\
k
\end{array}\right)\left[e^{\alpha b+\beta}-e^{\alpha a+\beta}\right],
\end{aligned}
$$

where $\alpha=p(r-2 k)$, and $\beta=a p(2 k-r)$.

Hence, the theorem follows.

Theorem 11. Let $f:[0, \infty) \rightarrow \mathbb{R}$ be a differentiable hyperbolic p-convex function, $n \in \mathbb{N}$, and $a, b \in[0, \infty]$ with $a<b$. Such that $f(0)>0, f^{\prime}(0)>0$. Then

$$
\int_{a}^{b} f^{2 n}(x) d x \geq \sum_{r=0}^{2 n}\left(\begin{array}{c}
2 n \\
r
\end{array}\right) f^{2 n-r}(0)\left(\frac{f^{\prime}(0)}{p}\right)^{r} \sum_{k=0}^{r} \frac{(-1)^{k}}{2^{r} \gamma}\left(\begin{array}{l}
r \\
k
\end{array}\right)\left[e^{\gamma b}-e^{\gamma a}\right],
$$

where, $\gamma=p(r-2 k)$.

Proof. Since, $f(x)$ is hyperbolic $p$-convex function, then from Definition 3 , we have

$$
f(x) \geq S_{0}(x) \forall x \in[0, \infty)
$$

As $f(0)>0$ and $f^{\prime}(0)>0$,

using Proposition 4, we conclude that $S_{0}(x)>0, \forall x \in[0, \infty)$ and consequently,

$$
f^{2 n}(x) \geq S_{0}^{2 n}(x) \forall n \in \mathbb{N}
$$

Thus, using (3) and $\cosh p x \geq 1$, one has

$$
\int_{a}^{b} f^{2 n}(x) d x \geq \int_{a}^{b} S_{0}^{2 n}(x) d x
$$




$$
\begin{aligned}
& =\int_{a}^{b}\left[f(0) \cosh p x+\frac{f^{\prime}(0)}{p} \sinh p x\right]^{2 n} d x \\
& =\sum_{r=0}^{2 n}\left(\begin{array}{c}
2 n \\
r
\end{array}\right) f^{2 n-r}(0)\left(\frac{f^{\prime}(0)}{p}\right)^{r} \int_{a}^{b} \cosh ^{2 n-r} p x \sinh ^{r} p x d x \\
& \geq \sum_{r=0}^{2 n}\left(\begin{array}{c}
2 n \\
r
\end{array}\right) f^{2 n-r}(0)\left(\frac{f^{\prime}(0)}{p}\right)^{r} \int_{a}^{b} \sinh ^{r} p x d x \\
& =\sum_{r=0}^{2 n}\left(\begin{array}{c}
2 n \\
r
\end{array}\right) f^{2 n-r}(0)\left(\frac{f^{\prime}(0)}{p}\right)^{r} \int_{a}^{b}\left[\frac{e^{p x}-e^{-p x}}{2}\right]^{r} d x \\
& =\sum_{r=0}^{2 n}\left(\begin{array}{c}
2 n \\
r
\end{array}\right) f^{2 n-r}(0)\left(\frac{f^{\prime}(0)}{p}\right)^{r} \sum_{k=0}^{r} \frac{(-1)^{k}}{2^{r}}\left(\begin{array}{l}
r \\
k
\end{array}\right) \int_{a}^{b} e^{p(r-k) x} e^{-p k x} d x \\
& =\sum_{r=0}^{2 n}\left(\begin{array}{c}
2 n \\
r
\end{array}\right) f^{2 n-r}(0)\left(\frac{f^{\prime}(0)}{p}\right)^{r} \sum_{k=0}^{r} \frac{(-1)^{k}}{2^{r}}\left(\begin{array}{l}
r \\
k
\end{array}\right) \int_{a}^{b} e^{p(r-2 k) x} d x \\
& =\sum_{r=0}^{2 n}\left(\begin{array}{c}
2 n \\
r
\end{array}\right) f^{2 n-r}(0)\left(\frac{f^{\prime}(0)}{p}\right)^{r} \sum_{k=0}^{r} \frac{(-1)^{k}}{2^{r} \gamma}\left(\begin{array}{l}
r \\
k
\end{array}\right)\left[e^{\gamma b}-e^{\gamma a}\right],
\end{aligned}
$$

where, $\gamma=p(r-2 k)$.

Hence, the theorem follows.

Theorem 12. Let $f:[0, \infty) \rightarrow \mathbb{R}$ be a non-negative differentiable hyperbolic $p$ convex function, $n \in \mathbb{N}$, and $a, b \in[0, \infty)$ with $a<b$. Such that $f^{\prime}(0)=0$, then has the following inequalities

$$
\begin{aligned}
\int_{a}^{b} f^{2 n}(x) d x & \geq\left(\frac{f(0)}{2}\right)^{2 n}\left[\left(\begin{array}{c}
2 n \\
n
\end{array}\right)(b-a)\right. \\
+ & \left.2 \sum_{r=0}^{n-1} \frac{1}{p(n-r)}\left(\begin{array}{c}
2 n \\
r
\end{array}\right) \cosh p(n-r)(b+a) \sinh p(n-r)(b-a)\right], \\
\int_{a}^{b} f^{2 n-1}(x) d x & \geq 4\left(\frac{f(0)}{2}\right)^{2 n-1} \sum_{r=0}^{n-1} \frac{2 n-1}{p r(2 n-2 r-1)} \cosh p\left(n-r-\frac{1}{2}\right)(b+a) \\
& \times \sinh p\left(n-r-\frac{1}{2}\right)(b-a) .
\end{aligned}
$$

Proof. Since, $f(x)$ is hyperbolic $p$-convex function, then from Definition 3 , we have

$$
f(x) \geq S_{0}(x) \forall x \in[0, \infty)
$$

Since, $f(x)$ is differentiable and $f^{\prime}(0)=0$, then from Proposition 4 the supporting function $S_{0}(x)$ for $f(x)$ at the point $0 \in[0, \infty)$ can be written in the form

$$
S_{0}(x)=f(0) \cosh p x .
$$


Hence, $S_{0}(x) \geq 0 \forall x \in[0, \infty)$ Thus, using (8), one obtains

$$
f^{n}(x) \geq S_{0}^{n}(x) \forall n \in \mathbb{N}
$$

Therefore, from (9) and (10), the following two cases arise,

Case 1.

$$
\begin{aligned}
\int_{a}^{b} f^{2 n}(x) d x & \geq \int_{a}^{b} S_{0}^{2 n}(x) d x \\
& =f^{2 n}(0) \int_{a}^{b} \cosh ^{2 n} p x d x \\
& =\left(\frac{f(0)}{2}\right)^{2 n} \int_{a}^{b}\left[\left(\begin{array}{c}
2 n \\
n
\end{array}\right)+\sum_{r=0}^{n-1} 2\left(\begin{array}{c}
2 n \\
r
\end{array}\right) \cosh 2 p(n-r) x\right] d x \\
& =\left(\frac{f(0)}{2}\right)^{2 n}\left[\left(\begin{array}{c}
2 n \\
n
\end{array}\right)(b-a)\right. \\
& \left.+2 \sum_{r=0}^{n-1} \frac{1}{p(n-r)}\left(\begin{array}{c}
2 n \\
r
\end{array}\right) \cosh p(n-r)(b+a) \sinh p(n-r)(b-a)\right]
\end{aligned}
$$

Case 2.

$$
\begin{aligned}
\int_{a}^{b} f^{2 n-1}(x) d x & \geq \int_{a}^{b} S_{0}^{2 n-1}(x) d x \\
& =f^{2 n-1}(0) \int_{a}^{b} \cosh ^{2 n-1} p x d x \\
& =2\left(\frac{f(0)}{2}\right)^{2 n-1} \int_{a}^{b} \sum_{r=0}^{n-1}\left(\begin{array}{c}
2 n-1 \\
r
\end{array}\right) \cosh p(2 n-2 r-1) x d x \\
& =4\left(\frac{f(0)}{2}\right)^{2 n-1} \sum_{r=0}^{n-1} \frac{2 n-1}{\operatorname{pr}(2 n-2 r-1)} \cosh p\left(n-r-\frac{1}{2}\right)(b+a) \\
& \times \sinh p\left(n-r-\frac{1}{2}\right)(b-a)
\end{aligned}
$$

Theorem 13. Let $f:[0, \infty) \rightarrow \mathbb{R}$ be an increasing differentiable hyperbolic $p$ convex function, $n \in \mathbb{N}$, and $a, b \in[0, \infty)$ with $a<b$. Such that $f(0)=0$, then has the following inequalities

$$
\begin{aligned}
\int_{a}^{b} f^{2 n}(x) d x & \geq\left(\frac{f(0)}{2}\right)^{2 n}\left[\left(\begin{array}{c}
2 n \\
n
\end{array}\right)(b-a)\right. \\
& \left.+2 \sum_{r=0}^{n-1} \frac{1}{p(n-r)}\left(\begin{array}{c}
2 n \\
r
\end{array}\right) \cosh p(n-r)(b+a) \sinh p(n-r)(b-a)\right]
\end{aligned}
$$




$$
\begin{aligned}
\int_{a}^{b} f^{2 n-1}(x) d x & \geq 4\left(\frac{f(0)}{2}\right)^{2 n-1} \sum_{r=0}^{n-1} \frac{1}{p(2 n-2 r-1)} \frac{2 n-1}{r} \cosh p\left(n-r-\frac{1}{2}\right) \\
& \times(b+a) \sinh p\left(n-r-\frac{1}{2}\right)(b-a) .
\end{aligned}
$$

Proof. Since, $f(x)$ is hyperbolic $p$-convex function, then from Definition 3 , we have

$$
f(x) \geq S_{0}(x) \forall x \in[0, \infty) .
$$

Since, $f(x)$ is increasing, then $f^{\prime}(0) \geq 0$. Since, $f(x)$ is differentiable and $f(0)=0$, then from Proposition 4, the supporting function $S_{0}(x)$ for $f(x)$ at the point $0 \in$ $[0, \infty)$ can be written in the form

$$
S_{0}(x)=\frac{f^{\prime}(0)}{p} \sinh p x .
$$

Hence, $S_{0}(x) \geq 0 \forall x \in[0, \infty)$ Thus, using (11), one obtains

$$
f^{n}(x) \geq S_{0}^{n}(x) \forall n \in \mathbb{N}
$$

Therefore, from (12) and (13), the following two cases arise, Case 1.

$$
\begin{aligned}
& \int_{a}^{b} f^{2 n}(x) d x \geq \int_{a}^{b} S_{0}^{2 n}(x) d x \\
& =\left(\frac{f^{\prime}(0)}{p}\right)^{2 n} \int_{a}^{b} \sinh ^{2 n} p x d x \\
& =\left(\frac{f^{\prime}(0)}{2 p}\right)^{2 n}(-1)^{n} \int_{a}^{b}\left[\left(\begin{array}{c}
2 n \\
n
\end{array}\right)+\sum_{r=0}^{n-1} 2(-1)^{n-r}\left(\begin{array}{c}
2 n \\
r
\end{array}\right) \cosh 2(n-r) p x\right] d x \\
& =\left(\frac{f^{\prime}(0)}{2 p}\right)^{2 n}(-1)^{n}\left[\left(\begin{array}{c}
2 n \\
n
\end{array}\right)(b-a)+4 \sum_{r=0}^{n-1} \frac{(-1)^{n-r}}{2 p(n-r)}\left(\begin{array}{c}
2 n \\
r
\end{array}\right) \cosh p(n-r)(b+a)\right. \\
& \times \sinh p(n-r)(b-a)] .
\end{aligned}
$$

Case 2.

$$
\begin{aligned}
& \int_{a}^{b} f^{2 n-1}(x) d x \geq \int_{a}^{b} S_{0}^{2 n-1}(x) d x \\
& =\left(\frac{f^{\prime}(0)}{p}\right)^{2 n-1} \int_{a}^{b} \sinh ^{2 n-1} p x d x \\
& =2\left(\frac{f^{\prime}(0)}{2 P}\right)^{2 n-1}(-1)^{n-1} \int_{a}^{b} \sum_{r=0}^{n-1}(-1)^{n+r-1}\left(\begin{array}{c}
2 n-1 \\
r
\end{array}\right) \sinh p(2 n-2 r-1) x d x \\
& =4\left(\frac{f(0)}{2}\right)^{2 n-1} \sum_{r=0}^{n-1} \frac{1}{p(2 n-2 r-1)} \frac{2 n-1}{r} \cosh p\left(n-r-\frac{1}{2}\right)
\end{aligned}
$$




$$
\times(b+a) \sinh p\left(n-r-\frac{1}{2}\right)(b-a) .
$$

Remark 14. For the hyperbolic expansions in Theorems 12, 13 one can refer to [11.

\section{Some applications For SPeCial Means}

Recall the following special means

(1) The arithmetic mean

$$
A=A(a, b):=\frac{a+b}{2}, \quad a, b \geq 0
$$

(2) The geometric mean

$$
G=G(a, b):=\sqrt{a b}, \quad a, b \geq 0
$$

(3) The harmonic mean

$$
H=H(a, b):=\frac{2 a b}{a+b}, \quad a, b \geq 0 ;
$$

(4) The Logarithmic mean

$$
L=L(a, b):=\frac{b-a}{\ln b-\ln a}, \quad a, b \geq 0, a \neq b ;
$$

(5) The Identic mean

$$
I=I(a, b)=\frac{1}{e}\left(\frac{b^{b}}{a^{a}}\right)^{\frac{1}{b-a}}, \quad a, b \geq 0, a \neq b ;
$$

(6) The m-Logarithmic mean

$$
L_{m}=L_{m}(a, b):=\left(\frac{b^{m+1}-a^{m+1}}{(m+1)(b-a)}\right)^{\frac{1}{m}}, a, b \geq 0, a \neq b ;
$$

where, $m \in \mathbb{R} \backslash\{-1,0\}$

it is well known that $L_{m}$ is monotonic nondecreasing over $m \in \mathbb{R}$ with $L_{-1}:=L$ and $L_{0}:=I$.

Proposition 15. Let $0<a<b$ and $m \in \mathbb{R} \backslash\{-1,0\}$. Then, we have the following inequality

$$
(b-a) L_{m}^{m}(a, b) \leq \sinh ^{-n} p(b-a) \sum_{r=0}^{n} \frac{1}{\mu}\left(\begin{array}{l}
n \\
r
\end{array}\right) a^{s(n-r)} b^{s r}\left[e^{\mu b+\lambda}-e^{\mu a+\lambda}\right],
$$

Proof. The assertion follows from inequality (6) in Theorem 9 for $f_{s}:(0, \infty) \rightarrow$ $(0, \infty), f_{s}(x)=x^{s}$ in Example 6 provided $[a, b] \subseteq\left(0, \frac{\sqrt{s(s-1)}}{|p|}\right), p \neq 0$ and $m=$ sn. 
Proposition 16. Let $0<a<b$ and $w \in \mathbb{R} \backslash\{-1,0\}$. Then we have the following inequality

$(b-a) L_{w}^{w}(a, b) \geq \sum_{r=0}^{2 n-1}\left(\begin{array}{c}2 n-1 \\ r\end{array}\right) a^{s(2 n-r-1)}\left(\frac{s a^{s-1}}{p}\right)^{r} \sum_{k=0}^{r} \frac{(-1)^{k}}{2^{r} \alpha}\left(\begin{array}{l}r \\ k\end{array}\right)\left[e^{\alpha b+\beta}-e^{\alpha a+\beta}\right]$,

Proof. The assertion follows from inequality 77 in Theorem 10 for $f_{s}:(0, \infty) \rightarrow$ $(0, \infty), f_{s}(x)=x^{s}$ in Example 6 provided $[a, b] \subseteq\left(0, \frac{\sqrt{s(s-1)}}{|p|}\right), p \neq 0$ and $w=$ $s(2 n-1)$.

\section{REFERENCES}

[1] Mohamed, S. S. A., On certain properties for two classes of generalized convex functions, Abstract and Applied Analysis, (2016), Article ID 4652038, 7 pages.

[2] Beckenbach, E. F., Generalized convex functions, Bulletin of the American Mathematical Society, vol. 43, no. 6, (1937), 363-371.

[3] Beckenbach, E. F. and Bing, R. H., On generalized convex functions, Transactions of the American Mathematical Society, vol. 58, (1945), 220-230.

[4] Peixoto, M. M., Generalized Convex Functions and Second Order Differential Inequalities, Bulletin of the American Mathematical Society, vol. 55, no. 6, (1949), 563-572.

[5] Toader, G. H., Some generalizations of the convexity, Proceedings of the Colloquium on Approximation and Optimization, University of Cluj-Napoca, (1984), 329-338.

[6] Hudzig, H., Maligranda, L., Some remarks ons-convex functions, Aequationes Mathematicae, vol. 48, no. 1, (1994), 100-111.

[7] Sarıkaya, M.Z., et al, Hermite-Hadamard's inequalities for fractional integrals and related fractional inequalities, Mathematical and Computer Modelling, vol. 57, no. 9, (2013), 24032407.

[8] Ion, D.A., Some estimates on the Hermite-Hadamard inequality through quasi-convex functions, Annals of the University of Craiova-Mathematics and Computer Science Series, vol. $34,(2007), 82-87$.

[9] Dragomir, S.S., On some new inequalities of Hermite-Hadamard type for $m$-convex functions, Tamkang Journal of Mathematics, vol. 33, no. 1, (2002), 45-56.

[10] Dragomir, S. S., Some inequalities of Hermite-Hadamard type for hyperbolic $p$-convex functions, RGMIA Res. Rep. Coll, 2018.

[11] Gradshteyn, I. S. and Ryzhik, I. M., Table of Integrals, Series and products, Academic Press, New York-London, 1983.

Current address: Nashat Faried: Department of Mathematics, Faculty of Science, Ain Shams University, Cairo, Egypt.

E-mail address: nashatfaried@sci.asu.edu.eg \& n_faried@hotmail.com

ORCID Address: http://orcid.org/0000-0002-9593-9699

Current address: Mohamed S. S. Ali: Department of Mathematics, Faculty of Education, Ain

Shams University, Cairo, Egypt.

E-mail address: mss_ali5@yahoo.com

ORCID Address: http://orcid.org/0000-0002-2219-6038

Current address: Zeinab M. Yehia: Department of Mathematics, Faculty of Education, Ain

Shams University, Cairo, Egypt.

E-mail address: zeinabyehia@edu.asu.edu.eg

ORCID Address: https://orcid.org/0000-0002-0263-9049 